\title{
Partially Mixed Duopoly and Oligopoly: Consistent Conjectural Variations Equilibrium (CCVE). Part 2.
}

\author{
Vyacheslav V. Kalashnikov ${ }^{1}$ Nataliya I. Kalashnykova $^{2}$ J. Fernando Camacho ${ }^{2}$ \\ ${ }^{1}$ ITESM, Campus Monterrey, Monterrey, N.L., Mexico \\ ${ }^{1}$ Central Economics and Mathematics Institute (CEMI), Moscow, Russia \\ ${ }^{1}$ Sumy State University, Sumy, Ukraine \\ ${ }^{2}$ FCFM, UANL, San Nicolás de los Garza, N.L., Mexico \\ kalash@itesm.mx, nkalash2009@gmail.com,fer_camachov@hotmail.com
}

\begin{abstract}
In this paper, a model of partially mixed oligopoly with conjectured variations equilibrium (CVE) is studied. The agents make conjectures concerning the price variations depending upon their production output's fluctuations. Existence and uniqueness results for the conjectured variations equilibrium (called an exterior equilibrium) is established for any collection of feasible conjectures. To deal with the concept of an interior (i.e., consistent) equilibrium, a consistency criterion for the influence coefficients is introduced and the existence theorem for the interior equilibrium (understood as a CVE with consistent conjectures) is established. As a base for the extension of the above-mentioned results to the case of non-differentiable demand functions, the behavior of the consistent conjectures as a function of a parameter representing the demand function's derivative with respect to the market price is investigated.
\end{abstract}

Keywords: Consistent Conjectural Variations Equilibrium, Partially Mixed Oligopoly.

\section{Introduction}

As it was stated in the introduction of Part 1 of this paper [1], here, in Part 2, we extend the results obtained in Part 1 for the partially mixed duopoly to the case of a partially mixed oligopoly. In the latter case, we also have a public company, which, like in Part 1, maximizes a convex combination of the net profit and domestic social surplus. However, in contrast to Part 1, here we have more than one of net profit maximizing subjects competing with the public company. Because of that, as strange it may sound, certain corresponding results are easier to proof than in the case of partially mixed duopoly.

The rest of Part 2 of the paper is organized as follows. Section 2 formulates the model and different types of equilibrium we consider (exterior and interior ones). In Section 3, we establish the main theorem of existence and uniqueness of the exterior equilibrium for any set of feasible conjectures (influence coefficients), as well as the formulas of the derivative of the equilibrium price $p$ with respect of the active demand variable $D$. Section 4 deals with the consistency criterion and the definition of an interior equilibrium (which can be treated as a consistent conjectural variations equilibrium, or CCVE); the CCVE existence theorem is also derived in that section. To provide the tools for the future research concerning the interrelationships between the demand structure (with not necessarily smooth demand function) and the conjectural variations equilibriums (CVEs) with consistent conjectures (influence coefficients), the behavior of the latter as functions of certain parameter governed by the derivative by $p$ of the demand function $G(p)$ is studied in Theorem 4.2 completing Section 4. Section 5 deals with an important particular case of a linear demand function. In Section 6, we conduct a qualitative analysis of results of the numerical experiments. Concluding remarks (Section 7), acknowledgments and the list of references complete the main body of the manuscript.

\section{Specification of the Oligopoly Model}

Consider a market with at least 3 producers of a homogeneous good with the cost functions $f_{i}\left(q_{i}\right), i=$ $0,1, \ldots, n, n \geq 2$, where $q_{i}$ is the output by producer $i$. Consumers' demand is described by a demand function $G(p)$, whose argument $p$ is the market price proposed by the producers. The value of an active demand $D$ is nonnegative and does not depend upon the price. We will fix the equilibrium between the demand and supply for a given price $p$ by the following balance equality

$$
\sum_{i=0}^{n} q_{i}=G(p)+D
$$


We assume the following properties of the model's data.

A1. The demand function $G=G(p)$ defined for the price values $p \in(0,+\infty)$ is non-increasing and continuously differentiable.

A2. For each agent $i=0,1, \ldots, n$, the cost function $f_{i}=f_{i}\left(q_{i}\right)$ is quadratic:

$$
f_{i}\left(q_{i}\right)=\frac{1}{2} a_{i} q_{i}^{2}+b_{i} q_{i},
$$

where $a_{i}>0, b_{i}>0, i=0,1, \ldots, n$. In addition, we assume that

$$
b_{0} \leq \max _{1 \leq i \leq n} b_{i}
$$

Remark 2.1 Although the assumption of $a_{i}>0$, $i=0,1, \ldots, n$, may look as inacceptable in view of the scale effect often observed in real-life production economy, it is not uncommon in theory of both classical and mixed oligopoly, see, e.g., [4] - [6], [22], to mention only few. In the majority of cases, this assumption is the easiest way to provide for the concavity of each player's payoff function. However, this condition can be somewhat relaxed, like, for example, in [23], where the second derivative of the cost function is not assumed to be positive. Then the desired payoff function's concavity is achieved by another assumption combining the first derivative of the demand function and the second derivative of the cost function. Finally, the scale effect can be also modeled by permitting the first order coefficients $b_{i}, i=0,1, \ldots, n$, to be negative. We have already obtained the corresponding results for this more general case, and they will be published elsewhere very soon.

Each private producer $i=1, \ldots, n$ chooses its output volume $q_{i} \geq 0$ so as to maximize its profit function $\pi_{i}\left(p, q_{i}\right)=p \cdot q_{i}-f_{i}\left(q_{i}\right)$. On the other hand, the public company with index $i=0$ produces $q_{0} \geq 0$ so as to maximize a convex combination of domestic social surplus (defined as the difference between the consumer surplus, the private company's total revenue, and the public firm's production costs):

$$
\begin{aligned}
& S\left(p ; q_{0}, \ldots, q_{n}\right)=\beta\left[\int_{0}^{\sum_{i=0}^{n} q_{i}} p(x) d x-\right. \\
& \left.-p\left(\sum_{i=1}^{n} q_{i}\right)-b_{0} q_{0}-\frac{1}{2} a_{0} q_{0}^{2}\right]+ \\
& +(1-\beta)\left(p q_{0}-b_{0} q_{0}-\frac{1}{2} a_{0} q_{0}^{2}\right)
\end{aligned}
$$

where $0<\beta \leq 1$. (Here, we follow the idea from [11] and [12].) Now we postulate that the agents (both public and private) assume that their choice of production volumes may affect the price value $p$. The latter assumption could be defined by a conjectured dependence of the price $p$ upon the output values $q_{i}$. If so, the first order maximum condition to describe the equilibrium would have the form: for the public company $(i=0)$

$$
\begin{gathered}
\frac{\partial S}{\partial q_{0}}=p-\left[(\beta-1) q_{0}+\beta q_{1}\right] \frac{\partial p}{\partial q_{0}}- \\
-f_{0}^{\prime}\left(q_{0}\right) \begin{cases}=0, & \text { if } q_{0}>0 \\
\leq 0, & \text { if } q_{0}=0 ;\end{cases}
\end{gathered}
$$

and for the private firm $i(i=1, \ldots, n)$

$$
\begin{gathered}
\frac{\partial \pi_{i}}{\partial q_{1}}=p+q_{i} \frac{\partial p}{\partial q_{i}}- \\
-f_{i}^{\prime}\left(q_{i}\right) \begin{cases}=0, & \text { if } q_{i}>0 \\
\leq 0, & \text { if } q_{i}=0 .\end{cases}
\end{gathered}
$$

Thus, we see that to describe the agent's behavior, we need evaluate the behavior of the derivative $\partial p / \partial q_{i}=-v_{i}$ rather than the dependence of $p$ upon $q_{i}$, $i=0,1, \ldots, n$. Here, we introduce the minus to deal with nonnegative values of $v_{i}$. Of course, the conjectured dependence of $p$ on $q_{i}$ must provide (at least the local) concavity of the $i$-th agent's conjectured profit as a function of its output.

For instance, it is enough to assume the coefficient $v_{i}$ (from now on referred to as the $i$-th agent's $i n$ fluence coefficient) to be nonnegative and constant. Then the conjectured local dependence of the profit upon the production output's variation $\eta_{i}$ has the form $\left[p-v_{i}\left(\eta_{i}-q_{i}\right)\right] \eta_{i}-f_{i}\left(\eta_{i}\right)$, while the maximum condition at $\eta_{i}=q_{i}, i=1, \ldots, n$, is provided by the relationships

$$
\begin{cases}p=v_{i} q_{i}+b_{1}+a_{i} q_{i}, & \text { if } q_{i}>0 \\ p \leq b_{i}, & \text { if } q_{i}=0 .\end{cases}
$$

Similarly, the public company conjectures the local dependence of domestic social surplus on its production output's variation $\eta_{0}$ in the form

$$
\begin{gathered}
\beta\left\{\int_{0}^{\eta_{0}+\sum_{i=1}^{n} q_{i}} p(x) d x-\left[p-v_{0}\left(\eta_{0}-q_{0}\right)\right] \times\right. \\
\left.\times \sum_{i=1}^{n} q_{i}\right\}+(1-\beta)\left[p-v_{0}\left(\eta_{0}-q_{0}\right)\right] \eta_{0}- \\
-f_{0}^{\prime}\left(\eta_{0}\right),
\end{gathered}
$$


which allows one to write down the maximum condition at $\eta_{0}=q_{0}$ as follows

$$
\begin{cases}p=-\beta v_{0} \sum_{i=1}^{n} q_{i}+ & \\ +(1-\beta) v_{0} q_{0}+b_{0}+a_{0} q_{0}, & \text { if } q_{0}>0 \\ p \leq-\beta v_{0} \sum_{i=1}^{n} q_{i}+b_{0}, & \text { if } q_{0}=0 .\end{cases}
$$

Were the agents' conjectures about the model given exogenously like it was assumed in [24] and [25], we would allow the values $v_{i}$ to be functions of $q_{i}$ and $p$. However, here we use the approach from papers [19] and [20], where the conjecture parameters for equilibrium are determined simultaneously with the price $p$ and the output values $q_{i}$ by a special verification procedure. In the latter case, the influence coefficients are the scalar parameters determined only for the equilibrium. In what follows, such equilibrium is referred to as interior one and is described by the set of variables and parameters $\left(p, q_{0}, \ldots, q_{n}, v_{0}, \ldots, v_{n}\right)$.

\section{Exterior Equilibrium in Oligopoly}

In order to present the verification procedure we need another notion of equilibrium called exterior ( $c f .$, [19] [21]) with parameters $v_{i}$ given exogenously. The set $\left(p, q_{0}, \ldots, q_{n}\right)$ is called an exterior equilibrium state for given influence coefficients $\left(v_{0}, \ldots, v_{n}\right)$, if the market is balanced, i.e., condition (1) is satisfied, and the maximum conditions (7) and (9) are valid.

In what follows, we are going to consider only the case when the set of really producing participants is fixed (i.e., it does not depend upon the values $v_{i}$ of the influence coefficients). To provide for this, we make the following assumption.

A3. For the price $p_{0}=\max _{1 \leq j \leq n} b_{j}$, the following estimate holds

$$
\sum_{i=0}^{n} \frac{p_{0}-b_{i}}{a_{i}}<G\left(p_{0}\right) .
$$

The latter assumption, together with assumptions $\mathbf{A} \mathbf{1}$ and $\mathbf{A 2}$, guarantees that for all nonnegative values of $v_{i}, i=1, \ldots, n$, and for $v_{0} \in\left[0, \bar{v}_{0}\right)$, where $\bar{v}_{0}>0$ and

$$
\bar{v}_{0}=a_{0}\left[\frac{G\left(p_{0}\right)-\frac{p_{0}-b_{0}}{a_{0}}}{\sum_{i=1}^{n} \frac{p_{0}-b_{i}}{a_{i}}}-1\right]
$$

if

$$
\sum_{i=1}^{n} \frac{p_{0}-b_{i}}{a_{i}}>0
$$

and $\bar{v}_{0}=+\infty$ otherwise, there always exists a unique solution of the optimality conditions (7) and (9) satisfying the balance equality (1), i.e., an exterior equilibrium state. Moreover, Theorem 3.2 will establish that conditions (1), (7) and (9) can hold simultaneously if, and only if $p>p_{0}$, that is, if and only if all outputs $q_{i}, i=$ $0, \ldots, n$, are strictly positive. The latter equivalence is demonstrated below.

Lemma 3.1. Let assumptions $\mathbf{A} \mathbf{1}-\mathbf{A} \mathbf{3}$ be valid. If a vector $\left(p, q_{0}, q_{1}, \ldots, q_{n}\right)$ is an exterior equilibrium state, then the relationship $p>p_{0}$ is equivalent to the fact that all $q_{i}>0, i=0,1, \ldots, n$.

Proof. If a vector $\left(p, q_{0}, q_{1}, \ldots, q_{n}\right)$ is an exterior equilibrium state then conditions (1), (7) and (9) hold simultaneously. In this case $p>p_{0}$ is equivalent to the fact that all outputs $q_{i}$ are strictly positive, $i=0,1, \ldots, n$. Indeed, if $p>p_{0}$ then it is evident that neither inequalities $p \leq b_{i}, i=1, \ldots, n$, from (7), nor $p \leq \beta v_{0} \sum_{i=1}^{n} q_{i}+b_{0}$ from (9) are possible, which means none of $q_{i}, i=0,1, \ldots, n$ can be zero. Conversely, if all $q_{i}>0, i=0,1, \ldots, n$, then it is straightforward from conditions (7) that

$$
p=v_{i} q_{i}+b_{i}+a_{i} q_{i}>b_{i}, \quad i=1, \ldots, n,
$$

hence $p>\max _{1 \leq j \leq n} b_{j}=p_{0}$.

Now we are in a position to formulate the main result of this section. We have proven the following theorem 3.2 and the details of the very long proofs can be provided by the authors upon request.

Theorem 3.2. Under assumptions A1, A2 and A3, for any $D \geq 0, v_{i} \geq 0, i=1, \ldots, n$, and $v_{0} \in$ $\left[0, \bar{v}_{0}\right)$, there exists uniquely an exterior equilibrium state $\left(p, q_{0}, q_{1}, \ldots, q_{n}\right)$ depending continuously upon the parameters $\left(D, v_{0}, v_{1}, \ldots, v_{n}\right)$. The equilibrium price $p=p\left(D, v_{0}, v_{1}, \ldots, v_{n}\right)$ as a function of these parameters is differentiable with respect to both $D$ and $v_{i}, i=0,1, \ldots, n$. Moreover, $p\left(D, v_{0}, v_{1}, \ldots, v_{n}\right)>p_{0}$, and

$$
\frac{\partial p}{\partial D}=\frac{1}{F(\beta, a, v, p)},
$$

with

$$
\begin{gathered}
F(\beta, a, v, p)=\frac{1}{a_{0}+(1-\beta) v_{0}}+ \\
+\frac{a_{0}+v_{0}}{a_{0}+(1-\beta) v_{0}} \sum_{j=0}^{n} \frac{1}{a_{j}+v_{j}}-G^{\prime}(p) .
\end{gathered}
$$

\section{Interior Equilibrium in Oligopoly}

Now we are ready to define an interior equilibrium state. To do that, we first describe the procedure of verification of the influence coefficients $v_{i}$ as it was given in [19] and [20]. Assume that we have an exterior equilibrium state $\left(p, q_{0}, q_{1}, \ldots, q_{n}\right)$ that has occurred for some $v_{0}, v_{1}, \ldots, v_{n}$, and $D$. One of the producers, 
say $k$, temporarily changes its behavior by abstaining from maximization of the conjectured profit (or domestic social surplus, as is in case $k=0$ ) and making small fluctuations around its output volume $q_{k}$. In mathematical terms, it is tantamount to restricting the model agents' list to the subset $\{i \neq k\}$ with the output $q_{k}$ subtracted from the active demand.

A fluctuation of the production output by agent $k$ is then equivalent to accepting the active demand varied as $D_{k}:=D-q_{k}$. If we consider these variations as infinitesimal, we suppose that by observing the corresponding variations of the equilibrium price, agent $k$ can estimate the derivative of the equilibrium price with respect to the active demand, which coincides with its own influence coefficient.

Applying formula (12) from Theorem 3.2 to calculate the derivatives, one has to remember that agent $k$ is (temporarily) excluded from the equilibrium model, hence one has to eliminate the terms with number $i=k$ from all the sums. Having that in mind, we come to the following criterion.

\subsection{Consistency Criterion}

At an exterior equilibrium state $\left(p, q_{0}, q_{1}, \ldots, q_{n}\right)$, the influence coefficients $\left(v_{0}, v_{1}, \ldots, v_{n}\right)$, are referred to as consistent if the following equalities hold:

$$
v_{0}=\frac{1}{\sum_{i=1}^{n} \frac{1}{a_{i}+v_{i}}-G^{\prime}(p)}
$$

and

$$
v_{i}=\frac{1}{\frac{a_{0}+v_{0}}{a_{0}+(1-\beta) v_{0}} \sum_{j \neq i} \frac{1}{a_{j}+v_{j}}-G^{\prime}(p)}
$$

Now we are ready to define the concept of an interior equilibrium state.

Definition $\quad$ 4.1. The collection $\left(p, q_{0}, q_{1}, \ldots, q_{n}, v_{0}, v_{1}, \ldots, v_{n}\right)$, where $v_{k} \geq 0, k=$ $0,1, \ldots, n$, is referred to as an interior equilibrium state if, for the considered influence coefficients, the collection $\left(p, q_{0}, q_{1}, \ldots, q_{n}\right)$ is an exterior equilibrium state, and the consistency criterion is satisfied for all $v_{k}, k=0,1, \ldots, n$.

Remark 4.2. If all the agents $i=0,1, \ldots, n$, were net profit-maximizing companies, equations (13) and (14) would be reduced to the uniform ones obtained independently in [20] and [22]:

$$
\begin{gathered}
v_{i}=\frac{1}{\sum_{j \neq i} \frac{1}{a_{j}+v_{j}}-G^{\prime}(p)} \\
i=0,1, \ldots, n
\end{gathered}
$$

The following theorem is an extension of Theorem 4.2 from [19] to the case of a partially mixed oligopoly.

Theorem 4.3. Under assumptions A1, A2 and A3, for any $D \geq 0$, there exists an interior equilibrium state.

Proof. The proof is an evident extension of that of Theorem 4.2 in [19].

In our future research, we are going to extend the obtained results to the case of non-differentiable demand functions. However, some of the necessary techniques can be developed now, in the differentiable case. To do that, we denote the value of the demand function's derivative by $\tau=G^{\prime}(p)$ and rewrite the consistency equations (13)-(14) in the following form:

$$
v_{0}=\frac{1}{\sum_{i=1}^{n} \frac{1}{a_{i}+v_{i}}-\tau}
$$

and

$$
\begin{gathered}
v_{i}=\frac{1}{\frac{a_{0}+v_{0}}{a_{0}+(1-\beta) v_{0}} \sum_{j \neq i} \frac{1}{a_{j}+v_{j}}-\tau}, \\
i=1, \ldots, n,
\end{gathered}
$$

where $\tau=[-\infty, 0]$. If $\tau=-\infty$ then system (16)-(17) has the unique solution $v_{i}(\tau)=0, i=0,1, \ldots, n$.

Theorem 4.4. For any $\tau \in(-\infty, 0]$ there exists a unique solution of equations (16)-(17), continuously depending upon $\tau$. Moreover, $v_{i}(\tau) \rightarrow 0$ when $\tau \rightarrow$ $-\infty, i=0,1, \ldots, n$, and $v_{0}(\tau)$ strictly increases up to $v_{i}(0)$ as $\tau$ grows and tends to zero, if

$$
\frac{n s}{n s+a_{0}(n-1)^{2}}<\beta \leq 1
$$

where $s=\max \left\{\bar{v}_{0}, a_{0}, a_{1}, \ldots, a_{n}\right\}$.

\section{A Special Case of Linear Demand Functions}

Let us consider a particular case of the linear demand function by introducing a new assumption instead of A1.

A4. The demand function is linear: $G(p)=-K p+T$ with $K>0, T>0$, and the ratio $T / K>0$ being large enough to provide that $G(p)>0$ for all possible equilibrium states that can happen in the model.

Now several interesting results concerning the behavior of the interior and exterior equilibria in dependence on the parameter $\beta \in(0,1]$ can be derived.

Theorem 5.1. For each $\beta \in(0,1]$, under assumptions A2-A4, there exists uniquely an interior equilibrium state $\left(p^{*}, q_{0}^{*}, q_{1}^{*}, \ldots, q_{n}^{*}, v_{0}^{*}, v_{1}^{*}, \ldots, v_{n}^{*}\right)=$ 
$=\left(p^{*}(\beta), q_{0}^{*}(\beta), q_{1}^{*}(\beta), \ldots, q_{n}^{*}(\beta)\right.$,

$\left.v_{0}^{*}(\beta), v_{1}^{*}(\beta), \ldots, v_{n}^{*}(\beta)\right)$. Moreover, the consistent coefficients of influence $v_{i}^{*}=v_{i}^{*}(\beta), i=0,1, \ldots, n$, treated as the (well-defined) functions of the variable $\beta$, are continuously differentiable over the feasible domain

$$
\frac{n s}{n s+a_{0}(n-1)^{2}}<\beta \leq 1,
$$

where $s=\max \left\{\bar{v}_{0}, a_{0}, a_{1}, \ldots, a_{n}\right\}$.

It is straightforward that the parameter $\beta$ can be interpreted as a measure of "privatization" of company $i=0(c f .,[12])$. Indeed, the smaller the value of $\beta$, the higher the relative weight of the net profit in the company's objective function (4). On the contrary, when $\beta \rightarrow 1$, the public company $i=0$ tends to behave more and more like the player maximizing domestic social surplus. Therefore, it is intuitively clear that when the parameter $\beta$ grows, the output produced by firm $i=0$ must go up, whereas the private companies $i=1, \ldots, n$, being downcast by the lowering price, should decrease its supply. Furthermore, it is also comprehensible that when $\beta$ grows, the total (passive) demand $G^{*}=G\left(p^{*}(\beta)\right)$ must increase, thus dropping the clearing (equilibrium) price $p^{*}(\beta)$. The latter evidently leads all the agents (private and public) of the market to the loss in their influence rates, i.e., the decrease in their influence coefficients $v_{i}^{*}(\beta)$, $i=0,1, \ldots, n$. For the particular case of the linear demand function, all these properties have been illustrated in the next section by the results of numerical experiments.

Remark 5.2. The obtained results allow one to conclude that starting from a certain "degree of socialization" $\bar{\beta} \in(0,1)$ achieved, the private companies are "crestfallen" and drop in both its production volumes $q_{i}$ and their self-evaluation parameters $v_{i}$. However, for the consumers, the growing of $\beta$ is the good news, since the total production volume increases, whereas the clearing price $p$, vice versa, goes down.

Remark 5.3. The threshold $\bar{\beta} \in(0,1)$ need not be tending to zero, as is shown by numerical experiments with the linear demand functions described in the next section.

\section{Numerical Experiments: Oligopoly}

To illustrate the difference between the partially mixed, mixed, and classical oligopoly cases related to the conjectural variations equilibrium with consistent conjectures (influence coefficients), we apply formulas (16)-(17) to a simple example of oligopoly in the electricity market from [19] and [22]. The only difference in our modified example from the instance of [22] is in the following: in their case, all six agents (suppliers) are private companies producing electricity and maximizing their net profits, and in our case, similar to [19], we assume that agent 0 (agent 5 in some instances) is a public enterprise seeking to maximize the convex combination of domestic social surplus and its profit described in (4), and the other generator is a private firm maximizing its net profit. On the other hand, similar numerical experiments were conducted and reported in [19] but only for $\beta=1$. All the other parameters involved in the inverse demand function $p=p(G, D)$ and the producers' cost functions, are exactly the same as in [22].

Therefore, following the above-mentioned references, we select the IEEE 2-generator 30-bus system $(c f .,[22])$ to illustrate our analysis. The inverse demand function in the electricity market is accepted to have the form:

$$
\begin{gathered}
p(G, D)=50-0.02(G+D)= \\
=50-0.02 \sum_{i=0}^{n} q_{i} .
\end{gathered}
$$

The cost functions parameters of suppliers (generators) are listed in Table 1 . Here, agents $0,1, \ldots 5$ will be combined in different examples listed below. In particular, Oligopoly 1 will involve agents 0 (public) and $1, \ldots, 5$ (private), whereas Oligopoly 2 comprises agents 5 (public) and $0,1, \ldots, 4$ (private).

Table 1: Cost functions' parameters

\begin{tabular}{|c|r|r|}
\hline Agent $i$ & $b_{i}$ & $a_{i}$ \\
\hline 0 & 2.00 & 0.02000 \\
\hline 1 & 1.75 & 0.01750 \\
\hline 2 & 3.00 & 0.02500 \\
\hline 3 & 3.00 & 0.02500 \\
\hline 4 & 1.00 & 0.06250 \\
\hline 5 & 3.25 & 0.00834 \\
\hline
\end{tabular}

To find the consistent influence coefficients in the classical oligopoly market (Case $1, \beta=0$ ), [22] uses formulas (15) for all six agents, while for the partially mixed oligopoly models (Oligopoly 1 or $2, \beta>0$ ), we exploit formulas (13) for the public agent (which is agent 0 in Oligopoly 1 , and agent 5 in Oligopoly 2) and (14) for the private companies (that is, 1 through 5 in Oligopoly 1 and 0 through 4 in Oligopoly 2), with $0<\beta<1$. Of course, when $\beta=1$, our model coincides with the mixed oligopoly studied in [19]. With thus obtained influence coefficients, the (unique) equilibrium is found for Oligopoly 1 and 2. The equilibrium results (influence coefficients, production outputs in MWh, equilibrium price, and the objective functions' optimal values in $\$$ per hour) are presented in Tables 2 through 9 . To make our conjectures $v_{i}$ comparable to those used in [21], [19], and [22], we divide 
them by $\left[-p^{\prime}(G)\right]=K^{-1}=0.02$ and thus come to $w_{i}:=-v_{i} / p^{\prime}(G)=K v_{i}=50 v_{i}, i=0,1, \ldots, n$, shown in Tables 2 and 6 , where columns Cournot and Perfect comprise the influence coefficients for the Cournot and the perfect competition models, respectively.

Next, Tables 3 through 5 demonstrate the numerical results for Oligopoly 1.

As Table 3 clearly reveals, the market clearing price (equilibrium price) in case of the classic duopoly $(\beta=$ $0)$ is $p_{1}=\$ 10.43$, which is higher than the mixed oligopoly equilibrium price $p_{2}=\$ 9.21$. The assertions of Remark 5.2 are also well-confirmed: the total production volume grows together with the public firm's output and domestic social surplus, while the clearing price (as well as the private companies' outputs and net profits) decrease when $\beta$ increases from 0 to 1 . A conclusion can be made: the higher the proportion of domestic social surplus in the public firm's objective, the greater the total production volume, hence, the lower the clearing price of electricity.

It is also interesting to compare the results in CVE with consistent conjectures against the production volumes and profits obtained for the same cases at the classic Cournot equilibrium (i.e., with all $w_{i}=1$, $i=0,1, \ldots, 5)$. Table 4 provides the numerical results, with $p_{3}=\$ 14.76$ in the classical duopoly $(\beta=0)$ much higher than the market equilibrium price $p_{4}=\$ 9.535$ in the mixed oligopoly $(\beta=1)$, the latter making only $67 \%$ of the former.

Again, the total electricity production level is monotone growing as the parameter $\beta$ increases starting from $G_{3}=1761.90 \mathrm{MWh}$ when $\beta=0$ and ending with $G_{4}=2023.256 \mathrm{MWh}$ for $\beta=1$. Another interesting observation can be made by comparing Tables 3 and 4: when $\beta$ is small $(\beta \leq 0.5)$, strong private companies (like agent 5) have higher objective functions' values by making use of the Cournot conjectures $w_{i}=1$, $i=0, \ldots, 5$. However, for $\beta$ greater than 0.5 , the orderings are converse: by relying on the consistent conjectures calculated by formulas (13)-(14) instead of the Cournot conjectures, the private companies improve their results significantly.

We also consider the perfect competition model (see Table 5) with $w_{i}=0, i=0, \ldots, 5$, which naturally gives the same results for all values of $\beta$ and uses to be the best for consumers. In our example, this model wins the mixed duopoly with consistent conjectures, both in the market price $p_{5}=\$ 8.97$ and in the total production volume $G_{5}=2051.57$ MWh. Domestic social surplus (with $\beta=1$ ) is also a bit higher in this case (of perfect competition), $\$ 43,303.52$ per hour, than that in the mixed oligopoly with consistent conjectures (also $\beta=1$ ), which is $\$ 42,187.80$ per hour.

Next, we estimate numerically the other model, namely, Oligopoly 2, where the private companies 0 through 4 compete with a much stronger public company 5 ( $c f$. ., Table 6.1 for its parameters). First, consistent coefficients of influence computed by (13)-(14) are shown in Table 6 below.

Now, Tables 7 through 9 demonstrate the numerical results for Oligopoly 2.

About Oligopoly 2 numerical results, similar comments may be formulated as for the previous Oligopoly 1. For instance, as Table 7 evidently demonstrates, the market clearing price (equilibrium price) in case of the classic oligopoly $(\beta=0)$ is quite elevated reaching $p_{6}=\$ 10.43$, in comparison to the mixed oligopoly equilibrium price $p_{7}=\$ 7.88$, which is almost $25 \%$ lower than the former one. The modes of behavior predicted by Remark 5.2 are confirmed, too: the total production volume grows together with the public firm's output and domestic social surplus, while the clearing price (as well as the private company's output and net profit) decreases when $\beta$ grows from 0 to 1 . Like in Oligopoly 1 , here, the same conclusion can be made: the higher the proportion of domestic social surplus in the public firm's objective fiunction, the greater the total production volume, hence, the lower the clearing price of electricity.

Again, it is worthy to compare the results in CVE with consistent conjectures versus the production volumes and profits obtained for the same cases at the classic Cournot equilibrium (i.e., with all $w_{i}=1$, $i=0,1, \ldots, 5)$. Table 8 presents the numerical results, with $p_{8}=\$ 14.76$ in the classical oligopoly $(\beta=0)$ essentially greater than the market equilibrium price $p_{9}=\$ 6.90$ in the mixed oligopoly $(\beta=1)$, which is only $40 \%$ of the former.

Similar to Oligopoly 1 , the total electricity production level is monotone growing as the parameter $\beta$ increases starting from $G_{6}=1978.5$ MWh when $\beta=0$ and ending with $G_{7}=2106.3 \mathrm{MWh}$ for $\beta=1$. A similar feature can found by comparing Tables 7 and 8: when $\beta$ is not too large $(\beta \leq 0.75)$, all companies have higher objective functions' values by making use of the 
Table 2: Coefficients of influence $w_{i}$ for Oligopoly 1

\begin{tabular}{|c|c|c|c|c|c|c|c|}
\hline Agent $i$ & $\beta=0$ & $\beta=0.25$ & $\beta=0.50$ & $\beta=0.75$ & $\beta=1$ & Cournot & Perfect \\
\hline 0 & 0.19275 & 0.19151 & 0.19027 & 0.18903 & 0.18779 & 1.0 & 0.0 \\
\hline 1 & 0.19635 & 0.18895 & 0.18155 & 0.17414 & 0.16574 & 1.0 & 0.0 \\
\hline 2 & 0.18759 & 0.18041 & 0.17323 & 0.16605 & 0.15887 & 1.0 & 0.0 \\
\hline 3 & 0.18759 & 0.18041 & 0.17323 & 0.16605 & 0.15887 & 1.0 & 0.0 \\
\hline 4 & 0.17472 & 0.16794 & 0.16117 & 0.15439 & 0.14761 & 1.0 & 0.0 \\
\hline 5 & 0.22391 & 0.21611 & 0.20831 & 0.20050 & 0.19270 & 1.0 & 0.0 \\
\hline
\end{tabular}

Table 3: Consistent equilibrium (production volumes $q_{i}$, the total volume $G$, price $p$, and the objective functions' values) for Oligopoly 1

\begin{tabular}{|c|r|r|r|r|r|}
\hline & $\beta=0$ & $\beta=0.25$ & $\beta=0.50$ & $\beta=0.75$ & $\beta=1$ \\
\hline$q_{0}$ & 353.405 & 421.555 & 489.706 & 557.856 & 626.006 \\
\hline$q_{1}$ & 405.120 & 393.375 & 381.629 & 369.883 & 358.138 \\
\hline$q_{2}$ & 258.436 & 248.940 & 239.444 & 229.947 & 220.451 \\
\hline$q_{3}$ & 258.436 & 248.940 & 239.444 & 229.947 & 220.451 \\
\hline$q_{4}$ & 142.898 & 138.539 & 134.180 & 129.821 & 125.462 \\
\hline$q_{5}$ & 560.180 & 542.361 & 524.543 & 506.723 & 488.905 \\
\hline$G$ & $1,978.5$ & $1,993.7$ & $2,008.9$ & $2,031.8$ & $2,039.4$ \\
\hline$p$ & 10.43 & 10.125 & 9.82 & 9.515 & 9.21 \\
\hline$S$ & $1,727.4$ & $11,842.5$ & $21,957.6$ & $32,072.7$ & $42,187.8$ \\
\hline$\pi_{1}$ & $2,076.6$ & $1,944.96$ & $1,813.32$ & $1,681.68$ & $1,550.04$ \\
\hline$\pi_{2}$ & $1,082.9$ & $1,002.65$ & 922.4 & 842.15 & 761.90 \\
\hline$\pi_{3}$ & $1,082.9$ & $1,002.65$ & 922.4 & 842.15 & 761.90 \\
\hline$\pi_{4}$ & 707.48 & 665.20 & 622.93 & 580.65 & 538.37 \\
\hline$\pi_{5}$ & $2,709.8$ & $2,511.85$ & $2,313.89$ & $2,115.93$ & $1,917.98$ \\
\hline
\end{tabular}

Cournot conjectures $w_{i}=1, i=0,1, \ldots, 5$. However, for $\beta$ greater than 0.75 , the orderings are converse: by relying on the consistent conjectures calculated by formulas (13)-(14) instead of the Cournot conjectures, all companies improve their results significantly.

We also consider the perfect competition model (see Table 9) with $w_{i}=0, i=0,1, \ldots, 5$, which naturally gives the same results for all values of $\beta$ and is known to be the best for consumers. However, in contrast to Oligopoly 1, in Oligopoly 2, the perfect competition results are runners-up to those of the mixed oligopoly with consistent conjectures, both in the market clearing price $p_{10}=\$ 8.97$ and in the total production volume $G_{8}=2051.6 \mathrm{MWh}$. In line with this, domestic social surplus (with $\beta=1$ ) is a bit lower in this case (of perfect competition), $\$ 44,050.7$ per hour, than that in the mixed oligopoly with consistent conjectures (also $\beta=1$ ), which is $\$ 44,477.3$ per hour.

Finally, by comparing pairwise Tables 3 and 7 , and Tables 4 and 8, we may see that the latter tables contain higher total production volumes and lower clearing prices than the former ones. These results may serve as a good example of how strong public companies may implicitly regulate the market price within a mixed oligopoly: the stronger the public company, the better results for the consumers.

Remark 6.1. The latter interesting feature may look counter-intuitive, since in the real life, it often happens that the stronger the private company, the higher the chance for monopoly and negative results for consumers. However, it may be so in the classical oligopoly but not in the mixed one. Indeed, it is easy to verify that the presence of a public company striving to maximize not its net profit but domestic social surplus completely excludes the possibility of the monopoly of any private company, no matter how strong it can be (cf., e.g., [19], where the public company never leaves the market, even if its net profit is negative and if it accepts the perfect competition conjecture $v_{0}=0$.) It is quite comprehensible, because the public company strives to maximize not its net profit but domestic social surplus. On the other hand, strong private companies can produce more than weak ones, thus decreasing the market clearing price, which is beneficial for the consumer.

\section{Concluding Remarks}

In this Part 2 of the paper, we consider a model of partially mixed oligopoly with Conjectural Variations Equilibrium (CVE). The agents' conjectures concern the price variations depending upon the increase or decrease of their production output. We establish the existence and uniqueness results for the conjectural 
Table 4: Cournot equilibrium (production volumes $q_{i}$, the total volume $G$, price $p$, and the objective functions' values) for Oligopoly 1

\begin{tabular}{|r|r|r|r|r|r|}
\hline & $\beta=0$ & $\beta=0.25$ & $\beta=0.50$ & $\beta=0.75$ & $\beta=1$ \\
\hline$q_{0}$ & 319.06 & 539.295 & 759.53 & 979.765 & $1,200.00$ \\
\hline$q_{1}$ & 347.00 & 312.15 & 277.30 & 242.45 & 207.60 \\
\hline$q_{2}$ & 261.39 & 232.35 & 203.31 & 174.26 & 145.22 \\
\hline$q_{3}$ & 261.39 & 232.35 & 203.31 & 174.26 & 145.22 \\
\hline$q_{4}$ & 166.82 & 150.98 & 135.14 & 119.29 & 103.45 \\
\hline$q_{5}$ & 406.23 & 360.11 & 314.00 & 267.88 & 221.77 \\
\hline$G$ & $1,761.9$ & $1,827.2$ & $1,892.6$ & $1,990.6$ & $2,023.3$ \\
\hline$p$ & 14.76 & 13.45 & 12.15 & 10.84 & 9.53 \\
\hline$S$ & $3,054.0$ & $11,184.9$ & $19,315.8$ & $27,446.6$ & $35,577.5$ \\
\hline$\pi_{1}$ & $3,461.7$ & $2,906.03$ & $2,350.36$ & $1,794.69$ & $1,239.02$ \\
\hline$\pi_{2}$ & $2,220.5$ & $1,836.72$ & $1,452.94$ & $1,069.16$ & 685.38 \\
\hline$\pi_{3}$ & $2,220.5$ & $1,836.72$ & $1,452.94$ & $1,069.16$ & 685.38 \\
\hline$\pi_{4}$ & $1,426.2$ & $1,206.78$ & 987.36 & 767.93 & 548.51 \\
\hline$\pi_{5}$ & $3,988.5$ & $3,288.55$ & $2,588.6$ & $1,888.65$ & $1,188.70$ \\
\hline
\end{tabular}

Table 5: Perfect competition equilibrium (production volumes $q_{i}$, the total volume $G$, price $p$, and the objective functions' values) for Oligopoly 1

\begin{tabular}{|r|r|r|r|r|r|}
\hline & $\beta=0$ & $\beta=0.25$ & $\beta=0.50$ & $\beta=0.75$ & $\beta=1$ \\
\hline$q_{0}$ & 348.43 & 348.43 & 348.43 & 348.43 & 348.43 \\
\hline$q_{1}$ & 412.49 & 412.49 & 412.49 & 412.49 & 412.49 \\
\hline$q_{2}$ & 238.74 & 238.74 & 238.74 & 238.74 & 238.74 \\
\hline$q_{3}$ & 238.74 & 238.74 & 238.74 & 238.74 & 238.74 \\
\hline$q_{4}$ & 127.50 & 127.50 & 127.50 & 127.50 & 127.50 \\
\hline$q_{5}$ & 685.68 & 685.68 & 685.68 & 685.68 & 685.68 \\
\hline$G$ & $2,051.6$ & $2,051.6$ & $2,051.6$ & $2,051.6$ & $2,051.6$ \\
\hline$p$ & 8.97 & 8.97 & 8.97 & 8.97 & 8.97 \\
\hline$S$ & $1,214.0$ & $11,736.4$ & $22,258.8$ & $32,781.1$ & $43,303.5$ \\
\hline$\pi_{1}$ & $1,488.80$ & $1,488.80$ & $1,488.80$ & $1,488.80$ & $1,488.80$ \\
\hline$\pi_{2}$ & 712.47 & 712.47 & 712.47 & 712.47 & 712.47 \\
\hline$\pi_{3}$ & 712.47 & 712.47 & 712.47 & 712.47 & 712.47 \\
\hline$\pi_{4}$ & 507.98 & 507.98 & 507.98 & 507.98 & 507.98 \\
\hline$\pi_{5}$ & $1,960.50$ & $1,960.50$ & $1,960.50$ & $1,960.50$ & $1,960.50$ \\
\hline
\end{tabular}

variations equilibrium (called an exterior equilibrium) for any set of feasible conjectures. To introduce the notion of an interior equilibrium state, we develop a consistency criterion for the conjectures (referred to as influence coefficients) and prove the existence theorem for the interior equilibrium (understood as a CVE with consistent conjectures).

To prepare the base for the extension of our results to the case of non-differentiable demand functions, we also investigate the behavior of the consistent conjectures in dependence upon a parameter representing the demand function's derivative with respect to the market price.

In our forthcoming papers, we are going to examine the qualitative behavior of prices and production outputs when the demand function is not necessarily differentiable, and the cost functions are not necessarily quadratic.

\section{Acknowledgment}

The research activity of the first author was financially supported by the R\&D Department (Cátedra de Investigación) CAT-174 of the Tecnológico de Monterrey (ITESM), Campus Monterrey, and by the SEP-CONACYT project CB-2008-01-106664, Mexico. Also, the work of the second and third authors was supported the National Counsel of Science and Technology (CONACyT) of Mexico as part of the project CB-2011-01-169765; PROMEP 103.5/11/4330, and PAICYT 464-10.

The authors would also like to express their profound gratitude to the two anonymous reviewers, whose critical but very constructive and valuable comments have helped the authors a lot to improve the presentation. 
Table 6: Coefficients of influence $w_{i}$ for Oligopoly 2

\begin{tabular}{|c|c|c|c|c|c|c|c|}
\hline Agent $i$ & $\beta=0$ & $\beta=0.25$ & $\beta=0.50$ & $\beta=0.75$ & $\beta=1$ & Cournot & Perfect \\
\hline 0 & 0.19275 & 0.1775 & 0.1624 & 0.1472 & 0.13208 & 1.0 & 0.0 \\
\hline 1 & 0.19635 & 0.1810 & 0.1656 & 0.1503 & 0.13497 & 1.0 & 0.0 \\
\hline 2 & 0.18759 & 0.1727 & 0.1578 & 0.1429 & 0.12803 & 1.0 & 0.0 \\
\hline 3 & 0.18759 & 0.1727 & 0.1578 & 0.1429 & 0.12803 & 1.0 & 0.0 \\
\hline 4 & 0.17472 & 0.1606 & 0.1465 & 0.1325 & 0.11843 & 1.0 & 0.0 \\
\hline 5 & 0.22391 & 0.2218 & 0.2198 & 0.2178 & 0.21584 & 1.0 & 0.0 \\
\hline
\end{tabular}

Table 7: Consistent equilibrium (production volumes $q_{i}$, the total volume $G$, price $p$, and the objective functions' values) for Oligopoly 2

\begin{tabular}{|r|r|r|r|r|r|}
\hline & $\beta=0$ & $\beta=0.25$ & $\beta=0.50$ & $\beta=0.75$ & $\beta=1$ \\
\hline$q_{0}$ & 353.405 & 329.924 & 306.443 & 282.961 & 259.480 \\
\hline$q_{1}$ & 405.120 & 379.647 & 354.175 & 328.702 & 303.229 \\
\hline$q_{2}$ & 258.436 & 238.048 & 217.660 & 197.272 & 176.884 \\
\hline$q_{3}$ & 258.436 & 238.048 & 217.660 & 197.272 & 176.884 \\
\hline$q_{4}$ & 142.898 & 133.670 & 124.441 & 115.213 & 105.984 \\
\hline$q_{5}$ & 560.180 & 691.081 & 821.983 & 952.884 & $1,083.785$ \\
\hline$G$ & $1,978.5$ & $2,010.4$ & $2,042.4$ & $2,090.3$ & $2,106.3$ \\
\hline$p$ & 10.43 & 9.79 & 9.15 & 8.51 & 7.88 \\
\hline$\pi_{0}$ & $1,727.40$ & $1,508.34$ & $1,289.28$ & $1,070.22$ & 851.16 \\
\hline$\pi_{1}$ & $2,076.6$ & $1,820.64$ & $1,564.68$ & $1,308.71$ & $1,052.75$ \\
\hline$\pi_{2}$ & $1,082.9$ & 929.98 & 777.06 & 624.14 & 471.22 \\
\hline$\pi_{3}$ & $1,082.9$ & 929.98 & 777.06 & 624.14 & 471.22 \\
\hline$\pi_{4}$ & 707.48 & 625.02 & 542.56 & 460.09 & 377.63 \\
\hline$S$ & $2,709.8$ & $13,151.7$ & $23,593.6$ & $34,035.4$ & $44,477.3$ \\
\hline
\end{tabular}

\section{References}

[1] V.V. Kalashnikov, N.I. Kalashnykova, and J.F. Camacho, Partially mixed duopoly and oligopoly: Consistent conjectural variations equilibrium (CCVE). Part 1, in print.

[2] R.C. Cornes and M. Sepahvand, Cournot vs Stackelberg equilibria with a public enterprise and international competition. Discussion Paper No.03/12, University of Nottingham, School of Economics, United Kingdom, 2003.

[3] C. Fershtman, The interdependence between ownership status and market structure: The case of privatization, Economica, 57:319-328, John Wiley \& Sons, 1990.

[4] T. Matsumura, Stackelberg mixed duopoly with a foreign competitor, Bulletin of Economics Research, 55:275-287, John Wiley \& Sons, 2003.

[5] N. Matsushima and T. Matsumura, Mixed oligopoly and spatial agglomeration, Canadian Journal of Economics, 36:62-87, John Wiley \& Sons, 2003.

[6] T. Matsumura and O. Kanda, Mixed oligopoly at free entry markets, Journal of Economics, 84:2748, Springer-Verlag, 2005.

[7] N.J. Ireland and P.J. Law, The Economics of Labour-Managed Enterprises, Croom Helm, Lon- don, 1982.

[8] J.P. Bonin and L. Putterman, Economics of Cooperation and the Labor-Managed Economy, Harwood Academic Publisher, Chur, Switzerland, 1987.

[9] F.H. Stephan (Ed.), The Performance of LabourManaged Firms, Macmillan Press, London, 1982.

[10] L. Putterman, Labour-managed firms. In S.N. Durlauf and L.E. Blume, editors, The New Palgrave Dictionary of Economics, vol. 4, pages 791795, Palgrave Macmillan, Basingstoke, Hampshire, 2008.

[11] B. Saha and R. Sensarma, State ownership, credit risk and bank competition: A mixed oligopoly approach. Working Paper, University of Hertfordshire Business School, Hatfield, England, 2009.

[12] A. Mumcu, S. Oğur, and Ü. Zenginobuz, Competition between regulated and non-regulated generators on electric power networks. MPRA Paper No. 376, online at http://mpra.ub.unimuenchen.de/376/ MPRA Paper No. 376, posted 07. November 2007 / 00:59.

[13] A.L. Bowley, The Mathematical Groundwork of Economics, Oxford University Press, Oxford, 1924.

[14] R. Frisch, Monopoly, polypoly: The concept of force in the economy, International Economics 
Table 8: Cournot equilibrium (production volumes $q_{i}$, the total volume $G$, price $p$, and the objective functions' values) for Oligopoly 2

\begin{tabular}{|r|r|r|r|r|r|}
\hline & $\beta=0$ & $\beta=0.25$ & $\beta=0.50$ & $\beta=0.75$ & $\beta=1$ \\
\hline$q_{0}$ & 319.06 & 269.95 & 220.84 & 171.72 & 122.61 \\
\hline$q_{1}$ & 347.00 & 294.61 & 242.23 & 189.84 & 137.45 \\
\hline$q_{2}$ & 261.39 & 217.73 & 174.08 & 130.42 & 86.77 \\
\hline$q_{3}$ & 261.39 & 217.73 & 174.08 & 130.42 & 86.77 \\
\hline$q_{4}$ & 166.82 & 143.01 & 119.19 & 95.38 & 71.57 \\
\hline$q_{5}$ & 406.23 & 717.08 & $1,027.92$ & $1,338.77$ & $1,649.61$ \\
\hline$G$ & $1,761.9$ & $1,860.1$ & $1,958.3$ & $2,105.7$ & $2,154.8$ \\
\hline$p$ & 14.76 & 12.80 & 10.83 & 8.87 & 6.90 \\
\hline$\pi_{0}$ & $3,054.0$ & $2,403.25$ & $1,752.51$ & $1,101.76$ & 451.01 \\
\hline$\pi_{1}$ & $3,461.7$ & $2,732.07$ & $2,002.44$ & $1,272.81$ & 543.18 \\
\hline$\pi_{2}$ & $2,220.5$ & $1,726.54$ & $1,232.59$ & 738.63 & 244.67 \\
\hline$\pi_{3}$ & $2,220.5$ & $1,726.54$ & $1,232.59$ & 738.63 & 244.67 \\
\hline$\pi_{4}$ & $1,426.2$ & $1,135.28$ & 844.36 & 553.43 & 262.51 \\
\hline$S$ & $3,988.5$ & $13,269.3$ & $22,550.0$ & $31,830.8$ & $41,111.6$ \\
\hline
\end{tabular}

Table 9: Perfect competition equilibrium (production volumes $q_{i}$, the total volume $G$, price $p$, and the objective functions' values) for Oligopoly 2

\begin{tabular}{|r|r|r|r|r|r|}
\hline & $\beta=0$ & $\beta=0.25$ & $\beta=0.50$ & $\beta=0.75$ & $\beta=1$ \\
\hline$q_{0}$ & 348.43 & 348.43 & 348.43 & 348.43 & 348.43 \\
\hline$q_{1}$ & 412.49 & 412.49 & 412.49 & 412.49 & 412.49 \\
\hline$q_{2}$ & 238.74 & 238.74 & 238.74 & 238.74 & 238.74 \\
\hline$q_{3}$ & 238.74 & 238.74 & 238.74 & 238.74 & 238.74 \\
\hline$q_{4}$ & 127.50 & 127.50 & 127.50 & 127.50 & 127.50 \\
\hline$q_{5}$ & 685.68 & 685.68 & 685.68 & 685.68 & 685.68 \\
\hline$G$ & $2,051.6$ & $2,051.6$ & $2,051.6$ & $2,051.6$ & $2,051.6$ \\
\hline$p$ & 8.97 & 8.97 & 8.97 & 8.97 & 8.97 \\
\hline$\pi_{0}$ & $1,214.0$ & $1,214.0$ & $1,214.0$ & $1,214.0$ & $1,214.0$ \\
\hline$\pi_{1}$ & $1,488.80$ & $1,488.80$ & $1,488.80$ & $1,488.80$ & $1,488.80$ \\
\hline$\pi_{2}$ & 712.47 & 712.47 & 712.47 & 712.47 & 712.47 \\
\hline$\pi_{3}$ & 712.47 & 712.47 & 712.47 & 712.47 & 712.47 \\
\hline$\pi_{4}$ & 507.98 & 507.98 & 507.98 & 507.98 & 507.98 \\
\hline$S$ & $1,960.50$ & $12,482.9$ & $23,005.27$ & $33,527.7$ & $44,050.0$ \\
\hline
\end{tabular}

Papers, 1:23-36, 1951. (Monopole, polypole - La notion de force en économie, Nationaløkonomisk Tidsskrift, 71:241-259, 1933.)

[15] J. Laitner, "Rational" duopoly equilibria, Quarterly Journal of Economics, 95:641-662, 1980.

[16] C. Figuières, A. Jean-Marie, N. Quérou, and M. Tidball, Theory of Conjectural Variations, World Scientific, Singapore, Taibei, 2004.

[17] N. Giocoli, The escape from conjectural variations: The consistency condition in duopoly theory from Bowley to Fellner, Cambridge Journal of Economics, 29:601-618, Oxford University Press, 2005.

[18] T. Lindh, The inconsistency of consistent conjectures. Coming back to Cournot, Journal of Economic Behavior and Organization, 18:69-90, Elsevier, 1992.

[19] V.V. Kalashnikov, V.A. Bulavsky, N.I. Kalash- nykova, and F.J. Castillo, Consistent conjectures in mixed oligopoly, European Journal of Operational Research, 210:729-735, Elsevier, 2011.

[20] V.A. Bulavsky, Structure of demand and equilibrium in a model of oligopoly, Economics and Mathematical Methods (Ekonomika i Matematicheskie Metody), 33:112-134, Central Economics and Mathematics Institute, Moscow, 1997 (in Russian).

[21] N.I. Kalashnykova, V.A. Bulavsky, V.V. Kalashnikov and F.J. Castillo-Pérez, Consistent conjectural variations equilibrium in a mixed duopoly, Journal of Advanced Computational Intelligence and Intelligent Informatics, 15:425-432, Fuji Technology Press, Japan, 2011.

[22] Y.F. Liu, Y.X. Ni, F.F. Wu, and B. Cai, Existence and uniqueness of consistent conjectural variation equilibrium in electricity markets, International 
Journal of Electrical Power and Energy Systems, 29:455-461, Elsevier, 2007.

[23] E.J. Dockner, A dynamic theory of conjectural variations, Journal of Industrial Economics, 40:377-395, 1992.

[24] V.A. Bulavsky and V.V. Kalashnikov, Oneparametric method to study equilibrium, Economics and Mathematical Methods (Ekonomika i Matematicheskie Metody), 30:129-138, Central Economics and Mathematics Institute, Moscow, 1994 (in Russian).

[25] V.A. Bulavsky and V.V. Kalashnikov, Equilibrium in generalized Cournot and Stackelberg models, Economics and Mathematical Methods (Ekonomika i Matematicheskie Metody), 31:164176, Central Economics and Mathematics Institute, Moscow, 1995 (in Russian). 